\title{
Evolutionary Framework for Multi-Dimensional Signaling Method Applied to Energy Dispatch Problems in Smart Grids
}

\author{
Fernando Lezama ${ }^{a}$, Enrique Munoz de Cote ${ }^{a, b}$, \\ Luis Enrique Sucar ${ }^{a}$ \\ ${ }^{a}$ Department of Computer Science, \\ Instituto Nacional de Astrofísica, Óptica \\ y Electrónica (INAOE), Mexico \\ ${ }^{b}$ PROWLER.io Ltd, Cambridge, United Kingdom \\ Email: \{f.lezama, jemc, esucar\}@inaoep.mx
}

\author{
João Soares, Zita Vale \\ GECAD-Research Group on Intelligent Engineering \\ and Computing for Advanced Innovation and Development \\ Polytechnic of Porto, Portugal \\ Email: \{joaps, zav\}@isep.ipp.pt
}

\begin{abstract}
In the smart grid (SG) era, the energy resource management (ERM) in power systems is facing an increase in complexity, mainly due to the high penetration of distributed resources, such as renewable energy and electric vehicles (EVs). Therefore, advanced control techniques and sophisticated planning tools are required to take advantage of the benefits that SG technologies can provide. In this paper, we introduce a new approach called multi-dimensional signaling evolutionary algorithm (MDS-EA) to solve the large-scale ERM problem in SGs. The proposed method uses the general framework from evolutionary algorithms (EAs), combined with a previously proposed rule-based mechanism called multi-dimensional signaling (MDS). In this way, the proposed MDS-EA evolves a population of solutions by modifying variables of interest identified during the evaluation process. Results show that the proposed method can reduce the complexity of metaheuristics implementation while achieving competitive solutions compared with EAs and deterministic approaches in acceptable times.
\end{abstract}

\section{INTRODUCTION}

The dawn of Smart Grids (SG) together with the high penetration of distributed generation (DG) possess a new level of complexity in power systems. The inclusion of Distribute Energy Resources (DER) and information and communication technologies (ICTs) in power grids promises potential benefits for both, network operators and users, as long as an efficient and effective management and control of DER can be achieved [1], [2].

One approach to allow high penetration of DER, while avoiding operational issues, is the aggregation of them through a virtual power plant (VPP). A VPP can provide same visibility controllability and market functionality as conventional generation by centralizing the management of DER in a common point [3]. However, to provide efficient operational support of VPPs in SGs, a complex Energy Resource Management (ERM) problem must be solved.

The ERM problem that a VPP must solve could consider a large variety of DER, such as Electric Vehicles (EVs), Energy Storage Systems (ESS), and DG including renewables [4]. Additionally, considering Demand Response (DR),
Vehicle-to-grid (V2G) capabilities, market bids and external suppliers, along with AC network power balance constraints turns the ERM into a Mixed-Integer Non-Linear Programming (MINLP) problem [5], [6]. Therefore, solving the large-scale ERM problem with deterministic approaches is a complex task that in some cases requires a huge amount of computational resources and time. In such situations, Evolutionary Algorithms (EAs) have proved to be a useful tool to addresses large-scale optimization problems, even when they cannot guarantee an optimal solution [7], [8].

In this paper, a new approach that combines the standard framework of EA with a knowledge-base method is proposed to solve the large-scale ERM in SGs. The Multi-Dimensional Signaling EA (MDS-EA) evolves a set of solutions using the standard framework of EAs, namely initialization, evaluation, generation of new solutions, and replacement. The proposed method uses a rule-based mechanism, initially introduced in [6], to cleverly identify variables of interest in the evaluation process. By doing so, the MDS-EA modifies the bounds of variables that directly affect the objective function value or lead to constraints violations, resulting in a more efficient exploration of the search space.

We compare the performance of the MDS-EA with some state-of-the-art population-based EAs, namely Differential Evolution (DE) [9], an application-specific modified Particle Swarm Optimization (ASMPSO) [10] and Differential Search Algorithm (DSA) [11]. Additionally, we provide the solution found with deterministic methods, such as MILP and MINLP, as a benchmark. Two case studies are used for the analysis, one corresponding to a 33-bus distribution network with DG and $1800 \mathrm{EVs}$, and a second considering a larger 180-bus distribution network with high penetration of DGs and 2000 EVs. Results show that a rule-based mechanism can boost the search capabilities of EAs and that MDS-EA can provide nearoptimal solutions for both case studies with simple mutation strategies. 


\section{Problem Formulation}

The day-ahead ERM model is based on a recent work [6], namely a multi-period optimization with 24 periods of 1 hour each. The ERM model includes the following assumptions: a) High accuracy models of load forecast, such as the ones developed in [12], [13], allows VPP predictions of the load with small errors. b) Advanced ICTs are available for monitoring and controlling of the grid guaranteeing optimal operation. c) The VPP can sell/buy energy to the main grid or other external entities. d) a centralized control approach is adopted in which a VPP aims to minimize its operational cost for the day-ahead (Eq. (1)) while maximizing its incomes (Eq. (2)). The total costs are modeled such as:

$$
\begin{aligned}
& \text { Minimize } \boldsymbol{O C}_{\text {Total }}^{\text {Day+1 }}= \\
& \sum_{t=1}^{T}\left(\begin{array}{l}
\left.\sum_{I=1}^{N_{I}} P_{D G(I, t)} \cdot C_{D G(I, t)}+\sum_{I=1}^{N_{I}} P_{G C P(I, t)} \cdot C_{G C P(I, t)}+\sum_{L=1}^{N_{L}} P_{N S D(L, t)} \cdot C_{N S D(L, t)}\right) \\
+\sum_{L=1}^{N_{L}} P_{L D R(L, t)} \cdot C_{L D R(L, t)}+\sum_{J=1}^{N_{J}} P_{\operatorname{Sup}(J, t)} \cdot C_{S u p(J, t)} \\
+\sum_{K=1}^{N_{K}} P_{\operatorname{Sdis}(K, t)} \cdot C_{\operatorname{Sdis}(K, t)}+\sum_{M=1}^{N_{M}} P_{V \operatorname{dis}(M, t)} \cdot C_{V \operatorname{dis}(M, t)}
\end{array}\right)
\end{aligned}
$$

where the first term corresponds to DG cost; the second term is the generation curtailment (GCP) cost; the third term refers to non-supplied demand (NSD) penalizations; the fourth term is the cost of DR programs; fifth term quantifies external suppliers energy cost; sixth and seventh terms are associated with discharging of EVs and ESS respectively. On the other hand, the total incomes are modeled as:

$$
\begin{aligned}
& \text { Maximize } \boldsymbol{I n}_{\text {Total }}^{\text {Day+1 }}= \\
& \sum_{t=1}^{T}\left(\begin{array}{l}
\sum_{L=1}^{N_{L}} P_{\operatorname{Load}(L, t)} \cdot U_{\operatorname{Load}(L, t)}+\sum_{N=1}^{N_{N}} P_{\operatorname{Sell}(N, t)} \cdot U_{\operatorname{Sell}(N, t)} \\
+\sum_{K=1}^{N_{K}} P_{S \operatorname{Sch}(K, t)} \cdot U_{\operatorname{Scha}(K, t)}+\sum_{M=1}^{N_{M}} P_{V \operatorname{Vha}(M, t)} \cdot U_{\operatorname{Vcha}(M, t)}
\end{array}\right)
\end{aligned}
$$

where the first term is the incomes obtained by selling energy; the second term is referred to profits of selling to the pool market; the fourth and fifth terms are the incomes that VPP perceives for charging EVs and ESS respectively.

Both equations (Eq. (1) and Eq. (2)) can be combined into a single equation as follows:

$$
\text { Minimize } f(\vec{x})=O C_{\text {Total }}^{\text {Day }+1}-\operatorname{In}_{\text {Total }}^{\text {Day } 1}
$$

where $f(\vec{x})$ represents the fitness function, and $\vec{x}$ is a solution, encoded in the vectorial space, including active and reactive power, and charge and discharge values for each DG, EV, and ESS, and for each period $t$. The full mathematical model also includes network power constraints, i.e. bus and line power limits. The reader can refer to [6] for details on the mathematical model, and to Appendix section for the notation of Eqs. (1-3).

\section{Multi-Dimensional Signaling Evolutionary ALGORITHM (MDS-EA)}

The proposed mechanism uses some ideas from EAs combined with a rule-based method, proposed in [6], called MultiDimensional Signaling (MDS). The original MDS (called embedded MDS in this paper) is a technique that improves the convergence capabilities of any population-based EA, e.g., PSO, DSA, or DE. Therefore, in the former work presented in [6], a particular EA is the main responsible for the search of the optimal solution, while MDS was somehow "embedded" inside of it to boost its search capabilities.

In this work, however, the MDS is used over a standard population-based EA framework. We call this approach MDSEA because it uses the common steps that characterize EAs, namely initialization, evaluation, generation of new solutions and selection, together with the MDS method of [6]. Figure 1 shows a flow chart of the MDS-EA. The main components of MDS are explained in the next subsections.

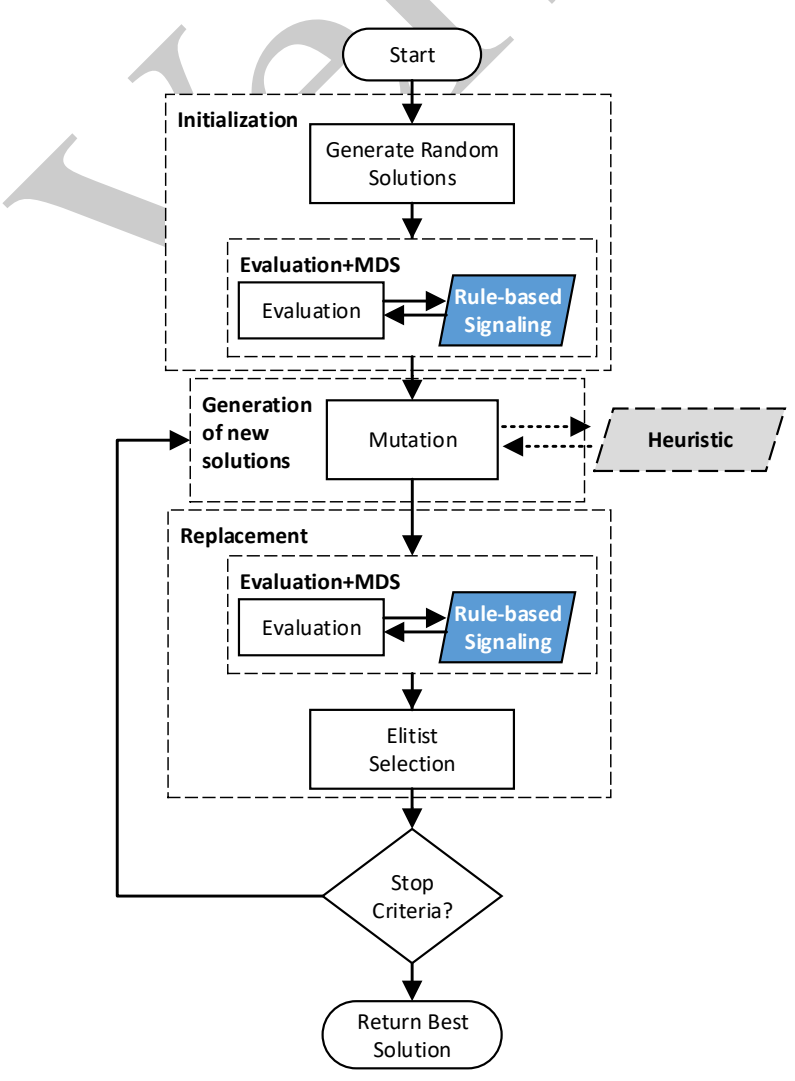

Fig. 1: MDS-EA flowchart.

\section{A. Initialization, Encoding of Solutions, and Evaluation}

A matrix $\operatorname{Pop}^{N \times D}$ with $N$ solutions and $D$ decision variables is defined to contain the population of solutions. Then, each solution is encoded as a vector $\vec{x}=\left[x_{1}, x_{2}, \ldots, x_{D}\right]$ where $D$ is the dimension of the problem. $\vec{x}$ for the ERM problem must contain the continuous values of active and reactive power within the limits of each DG. It also contains binary 
variables indicating a connection ('1' value) or disconnection ('0' value) of a particular DG. EVs and ESS variables can take negative and positive values within their ranges of discharge (negative value) and charge (positive value). All these values are encoded in $\vec{x}$ for each considered period $t$.

In the initialization stage, $N$ solutions are generated randomly within the range $\left[x_{l b, j}, x_{u b, j}\right]$, where $x_{l b, j}$ and $x_{u b, j}$ are the upper and lower bounds limits of the $j$ th variable respectively (i.e., the power limits of DGs, EVs and ESS, for each period $t$ ). After random initialization of Pop, all vectors are evaluated in the objective function. Different from other EAs, MDS-EA uses a rule-based mechanism (detailed in Sect. III-B) in the evaluation process to identify variables of interest.

\section{B. Multi-Dimensional Signaling}

MDS is a method proposed in [6] to improve metaheuristics' performance. The method is applied in the evaluation process to identify variables that can improve the value of the objective function or avoid constraints violation. After that, the method is applied to the main mutation operator of a metaheuristic to improve convergence capabilities. To do so, an MDS matrix is defined as $M^{N \times D} \in \mathbb{Z}^{N \times D}$ with $N$ rows corresponding to a number of solutions (e.g., population or number of particles in some EAs), and $D$ columns corresponding to the number of decision variables. The matrix $M$ change its value according to:

$$
M= \begin{cases}\operatorname{sig}_{a}(M), & \text { if } g\left(c_{a}\left(\vec{x}_{n}\right)\right) \geq 0, a \in A, n \in N \\ \operatorname{sig}_{b}(M), & \text { if cond }{ }_{b}=\text { true }, b \in \mathbb{N}, b \neq a \\ 0, & \text { otherwise }\end{cases}
$$

where $\operatorname{sig} F_{a}(M)$ and $\operatorname{sig} F_{b}(M)$ change the value of $M$ in each iteration. $A$ is the list of constraints that are suitable for signaling, $\operatorname{cond}_{b}$ is a custom condition $b$, and $\vec{x}_{n}$ is the solution $n \in N$. After identification of such variables, a modification in the bounds (i.e., a modification of the values that a variable can take) is applied, and MDS-EA is ready to apply any mutation strategy to generate new solutions.

Table I shows the rules applied to signaling variables during the evaluation process. In that table, $M C(t)$ corresponds to the marginal price of the system at time $t$. Signal code ' -1 ' indicates that signaled variables should take a negative value (i.e., EVs and ESS to discharge). On the contrary, signal code ' 1 ' indicates that signaled variables should take a positive value (i.e., EVs and ESS to charge). Finally, code '2' indicates that such variables should take a value of 0 (i.e., no participation of EVs and ESS, as well as DR and market sells).

\section{Mutation strategies}

Once the new bounds (or direction of movements) are defined, any mutation mechanism can be adopted over such signaled variables to create new solutions. In this paper, we explore the use of two simple generation strategies, namely MDS-EA with uniform mutation and MDS-EA with Gaussian mutation strategies.
TABLE I: Rules and signal codes applied during evaluation process.

\begin{tabular}{l|l|l} 
Variables & Rule & Signal Code \\
\hline \multirow{3}{*}{ Evs } & $M C(t) \geq C_{V \operatorname{dis}(M, t)}$ & -1 \\
& $M C(t) \leq U_{\operatorname{Vcha}(M, t)}$ & 1 \\
& Random & 2 \\
\hline \multirow{3}{*}{ ESS } & $M C(t) \geq C_{\operatorname{Sdis}(K, t)}$ & -1 \\
& $M C(t) \leq U_{\operatorname{Scha}(K, t)}$ & 1 \\
& Random & 2 \\
\hline \multirow{2}{*}{ DR } & $M C(t) \geq\left(C_{L D R(L, t)}+U_{\operatorname{Load}(L, t)}\right)$ & 1 \\
& $M C(t) \leq\left(C_{L D R(L, t)}+U_{\operatorname{Load}(L, t)}\right)$ & 2 \\
\hline \multirow{2}{*}{ Market } & $M C(t) \geq U_{\operatorname{sell}(N, t)}$ & 2 \\
& $M C(t) \leq U_{\operatorname{sell}(N, t)}$ & 1
\end{tabular}

In the first strategy, we create a new solution $\vec{m}$ as follows:

$$
\operatorname{NPop}_{i, j, G}= \begin{cases}x \sim U\left(\left[0, x_{u b, j}\right]\right) & \text { if } M_{i, j}=1 \\ x \sim U\left(\left[-x_{l b, j}, 0\right]\right) & \text { if } M_{i, j}=-1 \\ 0 & \text { if } M_{i, j}=2 \\ \operatorname{Pop}_{i, j, G} & \text { otherwise }\end{cases}
$$

where $N P \operatorname{Nop}_{i, j, G}$ is a new set of solutions created at generation $G$. Notice that Eq. (5) uses a uniform distribution to generate a random value for the variables signaled in matrix $M$. A probability $p_{r}$ can also be defined to control the application of the uniform distribution. If a random number rnd is less or equal to $p_{r}$ then the variable is set to 0 . Otherwise, the random generation is performed.

The second strategy includes a creation mechanism for the variables that are not signaled in the evolution process. Then a random number $x \sim N\left(\operatorname{Pop}_{i, j, G}, \sigma_{j}\right)$ is assigned to the entries of $N P o p_{i, j, G}$ that were not signaled in the evaluation process, instead of keeping the value of $\operatorname{Pop}_{i, j, G} . N\left(\operatorname{Pop}_{i, j, G}, \sigma_{j}\right)$ is a normal distribution with mean $\operatorname{Pop}_{i, j, G}$ and $\sigma_{j}=0.001 *$ $\left(x_{u b, j}-x_{l b, j}\right)$. This strategy then, allows flexibility in the exploration of variables that are not signaled in the evaluation process. More sophisticated mutation schemes can be explored and will be considered in future work.

\section{Elitist Replacement}

This operator is applied by comparing the fitness between the new set of solutions $N \operatorname{Pop}_{i, j, G}$ (originated with some of the above strategies), and the current $\operatorname{Pop}_{i, j, G}$ in the objective function. Then, $\operatorname{Pop}_{i, G+1}$ is the group of solutions of the next generation, that changes by accepting new individuals when they give a better fitness in the objective function (i.e., Eq. (4)). Notice that the signaling matrix $M$ will also be modified in this stage of evaluation, so even if an individual from $N P o p_{i, j, G}$ does not survive to the next generation, it changes matrix $M$ when its evaluation occurs giving new bounds for the creation of new solutions.

\section{RESUlTS AND Discussion}

In this section, the case studies and the application of MDSEA to the ERM problem are presented. All the experiments were performed using MATLAB 2014b 64 bits in a computer with an Intel Xeon W3565 processor and 6 GB of RAM 
running Windows 10 . The reported results correspond to the average of 30 test.

\section{A. Case Studies}

Two case studies, a $12.66 \mathrm{kV} 33$-bus and 30kV 180-bus distribution networks, are considered to test the MDS-EA ${ }^{1}$. Tables II and III present the resources for both case studies.

1) 33-bus Network: The 33-bus network scenario adapted from [16] includes 67 DGs (with a large wind unit), 10 external suppliers, 15 ESS and 1800 EVs with V2G capabilities. External suppliers are modeled as a substation connected to the main grid in bus 33. DR with Direct Load Control (DLC) is considered, setting DLC contracts to $0.16 \mathrm{~m} . \mathrm{u} . / \mathrm{kWh}$. The consumers receive this benefit for each unit of energy reduced, instead of paying the VPP contracted supply price of 0.14 m.u./kWh. The selling energy price is set to 0.14 m.u. $/ \mathrm{kWh}$ as well. A fleet of $1800 \mathrm{EVs}$ with V2G capabilities is considered with a total energy demand predicted for trips of $13.77 \mathrm{MWh}$ and a total of 2553 trips. The discharging cost for EVs and ESS is set to 0.19 m.u./kWh. The charging/discharging efficiency is set to $70 \%$ for EVs and $90 \%$ for ESS.

TABLE II: Available resources for 33-bus network [6]

\begin{tabular}{llll}
\hline Resources & No. Units & Price $(\mathbf{m . u} . / \mathbf{k W h})$ & Capacity $(\mathbf{k W})$ \\
\hline Biomass & 4 & 0.09 & 380 \\
CHP & 15 & 0.06 & 1150 \\
Fuel cell & 7 & 0.15 & 110 \\
Small hydro & 2 & 0.07 & 70 \\
PV & 31 & 0.2 & $0-840$ \\
Waste-to-energy & 1 & 0.1 & 10 \\
Wind & 6 & 0.15 & $180-890$ \\
Large wind & 1 & 0.07 & $1580-1800$ \\
External suppliers & 10 & $0.09-0.3$ & 6200 \\
V2G & 1800 & 0.19 & $0-5720$ \\
ESS & 15 & 0.19 & 900 \\
DR & 32 & 0.16 & $600-1170$ \\
\hline
\end{tabular}

2) 180-bus network: For this case study, a 6000 EVs fleet with total energy demand predicted of $34.26 \mathrm{MWh}$, corresponding on average to a $5.7 \mathrm{kWh}$ per vehicle, and a total of 10137 trips was considered. The discharging cost for the EVs was set to 0.19 m.u./kWh. For the ESS, the discharging cost (that also include battery degradation cost) was set to 0.18 m.u./kWh. Charging/discharging efficiency for both, EVs and ESS, was set to $90 \%$. External suppliers contracts are considered with a minimum purchase of $2 \mathrm{MW}$ in the considered time horizon and a maximum capacity of 10MW. Total forecast load without taking into account EVs, ESS and DR program was 243.36 MWh.

\section{B. Results and Comparison}

In this section, extensive experimentation showing the performance of MDS-EA, with uniform mutation and Gaussian mutation schemes, is presented. We also compare the results of MDS-EA with some popular EAs, namely DE (including

\footnotetext{
${ }^{1}$ Both networks represent a SG operated by a VPP with projections of DG and V2G penetration levels for the year 2040. The prices and capacities of DG take into account the observations made in [14]. The scenarios of EVs were developed using [15]. The complete data can be found in http://www.gecad.isep.ipp.pt/ies/public-data/swevo/.
}

TABLE III: Avaialable resources for 180-bus network [6]

\begin{tabular}{llll}
\hline Resources & Units & Price $(\mathbf{m . u} . / \mathbf{k W h})$ & Capacity (kWh) \\
\hline PV & 44 & 0.15 & 1490 \\
Wind & 55 & 0.09 & $1070-1760$ \\
Biomass & 17 & 0.13 & 1980 \\
External supplier & 1 & $0.10-0.16$ & 10000 \\
V2G & 6000 & 0.19 & 19050 \\
ESS & 7 & 0.18 & 1200 \\
DR & 90 & 0.16 & $2470-4260$
\end{tabular}

DE/rand/1 and DE/target-to-best/1 strategies) [9], DSA [11], and (ASMPSO) [10]. Results of the EAs with embedded MDS, as proposed in [6], are also presented. Also, two deterministic methods using MILP and MINLP are used as a benchmark.

All the population-base methods use a population of 10 solutions (e.g., individuals in DE or particles in PSO) and a stop criteria of either 400 iterations without objective function improvement or a total of 2000 iterations. All the implemented EAs use the same individual encoding in a vector space. An individual $\vec{x}$ includes decision variables that are within the limits of the active and reactive power of DG. EVs and ESS variables are negatives indicating a discharging power value, and positives otherwise (i.e., when charging). DR and external supplier are also included in individual $\vec{x}$ as positive continuous variables between allowed limits. Finally, binary variables indicating connection and disconnection of DGs are included as continuous values in the range $[0,1]$ and are evaluated using a rounding mechanism that maps such values to either ' 0 ' or ' 1 ' depending on their values. Regarding the particular set of parameters for each EA, for DE strategies an empirical configuration was carried out to determine the set of parameters that leads to good performance. Therefore, $F=0.3$ and $C r=0.5$ were used for DE/rand/1 strategy, while $F=0.8$ and $C r=0.4$ was adopted for DE/target-tobest/1 strategy. For DSA and ASMPSO, the parameter setting obtained with empirical experimentation in [6] was used.

Table IV presents the total income (IN), operational cost (OC), average profits (a negative fitness value represents positive profits because the algorithms were set up to minimize Eq. 4), time and number of iterations used by the methods for the two case studies. The table is divided into four horizontal sets. In the first set (first to fourth row), results of EAs without MDS are presented. In the second set (fifth to eighth row), we present the application of embedded MDS with the EAs as proposed in [6]. The third set (eighth and ninth rows), presents MDS-EA results with simple uniform and Gaussian strategies. Finally, the fourth set (eleventh and twelfth rows) presents the results with deterministic approaches as a benchmark.

It can be observed in the columns corresponding to case study 33-bus of Table IV, that ASMPSO presents the best performance among EAs without signaling. That was expected since ASMPSO is a PSO algorithm modified to tackle this specific problem in [10]. However, we observe that DE strategies and DSA still provide acceptable profits for this case study. In the second set of results, it is clear that the application of embedded MDS offers an improvement of fitness in all EAs. When MDS is embedded in the EAs, DE/rand/1 presents 
TABLE IV: 33-bus and 180-bus case studies results and comparison of methods.

\begin{tabular}{|c|c|c|c|c|c|c|c|c|c|c|}
\hline \multirow[b]{2}{*}{ Method } & \multicolumn{5}{|c|}{ 33-bus case study } & \multicolumn{5}{|c|}{ 180-bus case study } \\
\hline & IN (m.u.) & OC (m.u.) & Profits \pm std & Time & Iter & IN (m.u.) & OC (m.u.) & Profits \pm std & Time & Iter \\
\hline DE/rand/1 & 20552 & 17306 & $3247 \pm 73$ & 15 & 2000 & 47491 & 47345 & $146 \pm 57$ & 40 & 2000 \\
\hline DE/target-to-best/1 & 20741 & 17136 & $3605 \pm 50$ & 14 & 2000 & 47218 & 46870 & $347 \pm 53$ & 42 & 2000 \\
\hline DSA & 20762 & 17278 & $3484 \pm 97$ & 13 & 2000 & 47154 & 46935 & $219 \pm 97$ & 38 & 2000 \\
\hline ASMPSO & 16736 & 11522 & $5215 \pm 59$ & 15 & 1968 & 41647 & 39168 & $2479 \pm 17$ & 39 & 2000 \\
\hline DE/rand/1 + MDS & 16910 & 11363 & $5547 \pm \mathbf{1 0}$ & 14 & 1536 & 44939 & 41922 & $3018 \pm 10$ & 40 & 2000 \\
\hline DE/target-to-best/1 + MDS & 16860 & 11330 & $5530 \pm 11$ & 12 & 1609 & 44623 & 41627 & $2996 \pm 15$ & 38 & 1863 \\
\hline DSA + MDS & 16773 & 11234 & $5539 \pm 11$ & 12 & 1744 & 44983 & 41947 & $3036 \pm 12$ & 36 & 1986 \\
\hline PSO + MDS & 16736 & 11227 & $5510 \pm 12$ & 9 & 1169 & 44399 & 41473 & $2925 \pm 27$ & 29 & 1341 \\
\hline MDS + uniform strategy & 16765 & 11232 & $5533 \pm 9$ & 11 & 1554 & 45056 & 42014 & $3042 \pm 6$ & 34 & 1948 \\
\hline MDS + Gaussian strategy & 16799 & 11263 & $5536 \pm 8$ & 12 & 1696 & 44973 & 41932 & $3041 \pm 7$ & 37 & 2000 \\
\hline MILP & 17110 & 11256 & $5854 \pm-$ & 0.2 & - & 42571 & 38813 & $3758 \pm-$ & 0 & - \\
\hline MINLP & 16968 & 11301 & $5667 \pm-$ & 834.3 & - & 42303 & 38938 & $3365 \pm-$ & 0 & \\
\hline
\end{tabular}

the best performance (showed in bold). Overall, our MDSEA method provides competitive results compared with the other EAs in terms of profits. It is worth noting that MDSEA and EAs+embedded MDS present competitive results compared with the deterministic approaches. MILP approach returns a solution in only 0.2 minutes, but such method does not consider the full mathematical model (including network constraints), which can lead to solutions that violate voltage and thermal limits in the lines (i.e., unfeasible solutions). MINLP and EAs consider the full model during their optimization process. However, MINLP returns the optimal solution in approximately 834 minutes, while EAs methods return acceptable solutions in 13 minutes on average.

The behavior of EAs and MDS-EA is similar when such methods are applied to the 180-bus case study. In this case, the EAs without MDS (columns of 180-bus case study in Table IV) present very poor performance regarding profits (except for ASMPSO, that got 2479 of profits). When MDS is embedded, the metaheuristics improve their performances. Among them, MDS-EA with uniform mutation gives slightly better profits compared with all EAs. However, MDS-EA is still 300 m.u. below the optimal solution found with MINLP, so a window for improvement is still open. Moreover, the time required to find the optimal solution with the MINLP was around 19361 minutes (more than 13 days), while the EAs return a solution in around 37 minutes.

Figure 2 shows box plots (including minimum, 25th, median, 75th and maximum values) for the average results

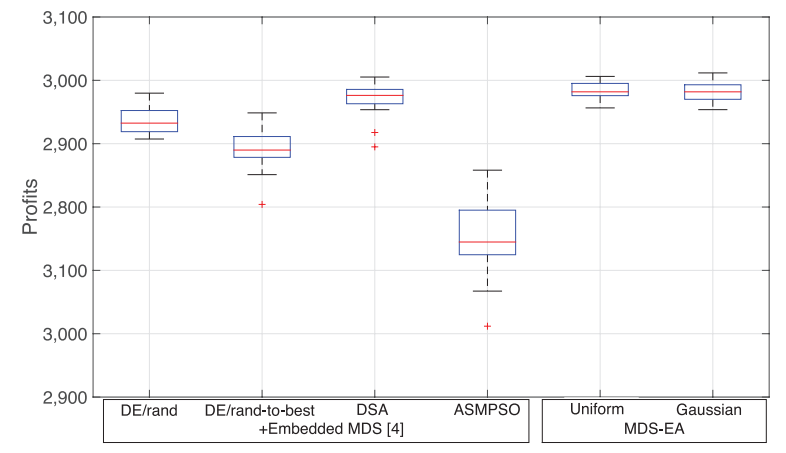

Fig. 2: Box plot for results in 180-bus network. of EAs+embedded MDS and MDS-EA with uniform and Gaussian strategies applied to the 180-bus network. It can be noticed that MDS-EA, with both uniform and Gaussian mutation, presents the best performance regarding average profits. The performance of MDS-EA is more robust, compared with the other EAs tested, since MDS-EA presents less variability in the results. ASMPSO and DE/target-to-best/1 with embedded MDS present the worst performance, with average profits below the $3 \mathrm{k}$. All the other heuristics show profits higher than the $3 \mathrm{k}$. The profits of the implemented EAs are still far from the optimal result found with the MILP approach (without considering full network constraints). Compared with the MINLP, EAs obtained a value around 300 m.u. below the optimal, which is an acceptable solution concerning m.u..

Finally, Fig. 3 shows the best scheduling obtained with MDS-EA with a uniform strategy for the 180-bus network case study. It can be observed that EVs have a substantial charging activity during the late night. This activity may be due to EVs should guarantee a good charge capacity to make the trips during the day. The scheduling also present a high participation of external suppliers, mainly due to an excessive amount of load to supplied and a low DG generation in all the periods. DR programs are used in periods $12-13$ and 19-20, which corresponds commonly to peak hours of the day, so the solutions make an adequate use of this feature.
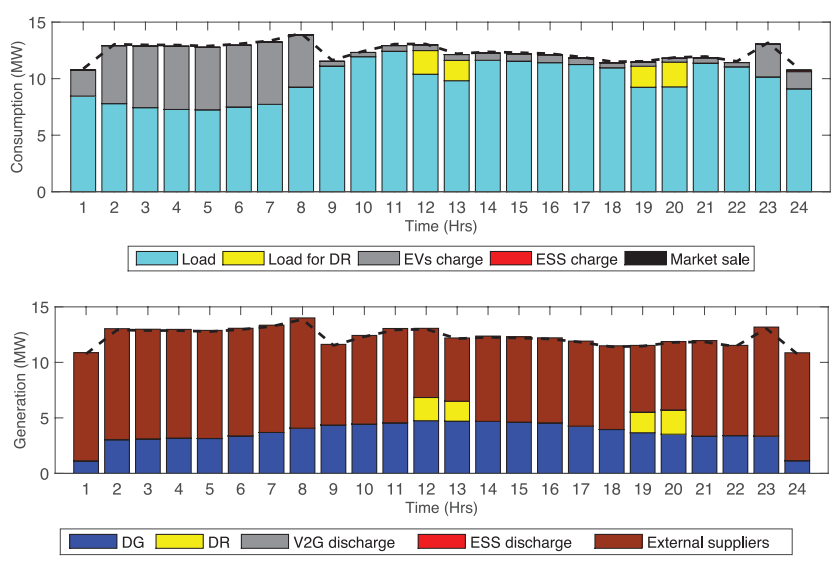

Fig. 3: MDS-EA consumption and generation scheduling. 


\section{CONCLUSIONS}

In this paper, a new approach called MDS-EA was proposed to solve the large-scale ERM problem in SGs. The MDS-EA combines the standard framework of population-based EAs (i.e., initialization, evaluation, generation of new solutions, and selection) with a rule-based technique, called MDS, that was originally proposed to work as an embedded method for population-based metaheuristics. Results show that MDSEA with a simple uniform or Gaussian mutation strategy can provide competitive and more robust results compared with other well-known EAs. Moreover, MDS-EA uses a simple framework that eases the implementation and complexity of the algorithm compared with sophisticated heuristics such as DSA+MDS or ASMPSO+MDS. As future work, more advanced generation strategies, based e.g. in local search rather than uniform and Gaussian random generation presented in this work, can be explored to improve the performance of the MDS-EA. Also, MDS-EA relies on a proper design of the set of rules employed in the domain of application, so to explore a new design of rules or automatic identification of them are other interesting research directions for further work.

\section{APPENDIX}

$\begin{array}{ll}\text { Indices: } & \\ t & \text { period } \\ I & \text { DG units } \\ L & \text { loads } \\ J & \text { external suppliers } \\ K & \text { ESS } \\ M & \text { EVs } \\ N & \text { energy buyers }\end{array}$

\section{Parameters:}

$T$ $N_{I}$

$N_{L}$

$N_{J}$

$N_{K}$

$N_{M}$

$C_{D G(I, t)}$

$C_{G C P(I, t)}$

$C_{N S D(L, t)}$

$C_{L D R(L, t)}$

$C_{\operatorname{Sup}(J, t)}$

$C_{S d i s(K, t)}$

$C_{V \operatorname{dis}(M, t)}$

$U_{\text {Load }(L, t)}$

$U_{\text {Sell }(N, t)}$

$U_{S c h a(K, t)}$

$U_{V c h a(M, t)}$

$P_{\text {Load }(L, t)}$

Variables:

$O C_{\text {Total }}^{\text {Day }+1}$

In ${ }_{\text {Total }}^{\text {Dat }}$

$P_{D G(I, t)}$

$P_{G C P(I, t)}$

$P_{N S D(L, t)}$

$P_{L D R(L, t)}$

$P_{\text {Sup }(J, t)}$

$P_{\text {Sdis }(K, t)}$

$P_{V \operatorname{dis}(M, t)}$

$P_{\text {Sell }(N, t)}$

$P_{\text {Scha }(K, t)}$

$P_{\operatorname{Vcha}(M, t)}$ number of DG number of loads number of external suppliers number of ESS number of EVs generation cost of DG $I$ in period $t$ (m.u. $/ \mathrm{kWh}$ ) generation curtailment cost of DG $I$ in period $t$ (m.u. $/ \mathrm{kWh}$ ) non-supplied demand cost of load $L$ in period $t$ (m.u./kWh) demand response cost of load $L$ in period $t$ (m.u. $/ \mathrm{kWh}$ ) energy price of external supplier $J$ in period $t$ (m.u./kWh) discharging cost of ESS $K$ in period $t$ (m.u. $/ \mathrm{kWh})$ discharging cost of EV $M$ in period $t$ (m.u. $/ \mathrm{kWh}$ ) electricity retail price of load $L$ in period $t$ (m.u./kWh) electricity sell price to market $N$ in period $t$ (m.u./kWh) charging price of ESS $K$ in period $t$ (m.u. $/ \mathrm{kWh}$ ) charging price of EV $M$ in period $t$ (m.u. $/ \mathrm{kWh}$ ) day-ahead active power forecast of load $L$ in $t(\mathrm{~kW})$

\section{total day-ahead operation cost (m.u.)}

total day-ahead income (m.u.)

active power generation of DG $I$ in period $t(\mathrm{~kW})$ generation curtailment power of DG $I$ in period $t(\mathrm{~kW})$ non-supplied demand power of load $L$ in period $t(\mathrm{~kW})$ active power reduction of load $L$ in period $t(\mathrm{~kW})$ active power flow in the branch connecting to external supplier $J$ in period $t(\mathrm{~kW})$

Power discharge of EES $K$ in period $t(\mathrm{~kW})$

Power discharge cost of EV $M$ in period $t$ (kW.)

electricity sell price to market $N$ in period $t(\mathrm{~kW})$

Power charge of EES $K$ in period $t(\mathrm{~kW})$

Power charge of EV $M$ in period $t(\mathrm{~kW})$

\section{number of periods}

\section{ACKNOWLEDGMENT}

The present work was done and funded in the scope of the projects: Project NetEffiCity (ANI-P2020 18015), and from FEDER Funds through COMPETE program and from National Funds through FCT under the project UID/EEA/00760/2013; Sustainability Fund CONACYT-SENER by Consejo Nacional de Ciencia y Tecnología (CONACYT) and the National Center of Innovation in Energy (CEMIE-Eolico, Project No. 206842).

\section{REFERENCES}

[1] J. I. Moreno, M. Martínez-Ramón, P. S. Moura, J. Matanza, and G. López, "Smart grid: ICT control for distributed energy resources," International Journal of Distributed Sensor Networks, vol. 12, no. 5, pp. ID-1 329421, 2016.

[2] F. Lezama, J. Palominos, A. Y. Rodríguez-González, A. Farinelli, and E. Munoz de Cote, "Agent-based microgrid scheduling: An ICT perspective," Mobile Networks and Applications, pp. 1-17, 2017.

[3] P. Faria, T. Soares, Z. Vale, and H. Morais, "Distributed generation and demand response dispatch for a virtual power player energy and reserve provision," Renewable Energy, vol. 66, pp. 686-695, 2014.

[4] Y. Yan, Y. Qian, H. Sharif, and D. Tipper, "A survey on smart grid communication infrastructures: Motivations, requirements and challenges," IEEE communications surveys \& tutorials, vol. 15, no. 1, pp. 5-20, 2013.

[5] A. Trivedi, D. Srinivasan, S. Biswas, and T. Reindl, "A genetic algorithm - differential evolution based hybrid framework: Case study on unit commitment scheduling problem," Information Sciences, vol. 354, pp. $275-300,2016$

[6] J. Soares, M. A. F. Ghazvini, M. Silva, and Z. Vale, "Multi-dimensional signaling method for population-based metaheuristics: Solving the largescale scheduling problem in smart grids," Swarm and Evolutionary Computation, vol. 29, pp. 13 - 32, 2016.

[7] A. Trivedi, D. Srinivasan, S. Biswas, and T. Reindl, "Hybridizing genetic algorithm with differential evolution for solving the unit commitment scheduling problem," Swarm and Evolutionary Computation, vol. 23, pp. 50-64, 2015.

[8] F. Lezama, G. Castañón, A. M. Sarmiento, and I. B. Martins, "Differential evolution optimization applied to the routing and spectrum allocation problem in flexgrid optical networks," Photonic Network Communications, vol. 31, no. 1, pp. 129-146, 2016.

[9] S. Das, S. S. Mullick, and P. Suganthan, "Recent advances in differential evolution - an updated survey," Swarm and Evolutionary Computation, vol. 27 , pp. $1-30,2016$.

[10] J. Soares, T. Sousa, H. Morais, Z. Vale, B. Canizes, and A. Silva, "Application-specific modified particle swarm optimization for energy resource scheduling considering vehicle-to-grid," Applied Soft Computing, vol. 13, no. 11, pp. $4264-4280,2013$.

[11] P. Civicioglu, "Transforming geocentric cartesian coordinates to geodetic coordinates by using differential search algorithm," Computers \& Geosciences, vol. 46, pp. 229-247, 2012.

[12] M. Motevasel and A. R. Seifi, "Expert energy management of a microgrid considering wind energy uncertainty," Energy Conversion and Management, vol. 83, pp. 58-72, 2014.

[13] W. Su, J. Wang, and J. Roh, "Stochastic energy scheduling in microgrids with intermittent renewable energy resources," IEEE Transactions on Smart Grid, vol. 5, no. 4, pp. 1876-1883, 2014.

[14] A. Zervos, C. Lins, and J. Muth, RE-thinking 2050: a 100\% renewable energy vision for the European Union. EREC, 2010.

[15] J. Soares, B. Canizes, C. Lobo, Z. Vale, and H. Morais, "Electric vehicle scenario simulator tool for smart grid operators," Energies, vol. 5, no. 6, pp. 1881-1899, 2012.

[16] M. E. Baran and F. F. Wu, "Network reconfiguration in distribution systems for loss reduction and load balancing," IEEE Transactions on Power Delivery, vol. 4, no. 2, pp. 1401-1407, Apr 1989. 